\title{
A Theory of Information Trilogy: Digital Ecosystem Information Exchange Architecture
}

\author{
Asif Qumer Gill (D)
}

Citation: Gill, A.Q. A Theory of Information Trilogy: Digital Ecosystem Information Exchange Architecture. Information 2021, 12, 283. https://doi.org/10.3390/ info12070283

Academic Editor: Arkaitz Zubiaga

Received: 20 June 2021

Accepted: 15 July 2021

Published: 16 July 2021

Publisher's Note: MDPI stays neutral with regard to jurisdictional claims in published maps and institutional affiliations.

Copyright: (C) 2021 by the author. Licensee MDPI, Basel, Switzerland. This article is an open access article distributed under the terms and conditions of the Creative Commons Attribution (CC BY) license (https:// creativecommons.org/licenses/by/ $4.0 /)$.
School of Computer Science, University of Technology Sydney, Ultimo, NSW 2007, Australia; asif.gill@uts.edu.au; Tel.: +61-2-9514-7938

\begin{abstract}
Information sharing is a critical component of a distributed and multi-actor digital ecosystem (DE). DE actors, individuals and organisations, require seamless, effective, efficient, and secure architecture for exchanging information. Traditional point-to-point and ad hoc integrations hinder the ability of DE actors to do so. The challenge is figuring out how to enable information sharing in a complex DE. This paper addresses this important research challenge and proposes the theory of information trilogy and conceptual DE information exchange architecture, which is inspired by the study of nature and flow of matter, energy, and its states in natural ecosystems. This work is a part of the large DE information framework. The scope of this paper is limited to the emerging concept of $\mathrm{DE}$ information exchange. The application of the DE information exchange concept is demonstrated with the help of a geospatial information sharing case study example. The results from this paper can be used by researchers and practitioners for defining the DE information exchange as appropriate to their context. This work also complements Shannon's mathematical theory of communication.
\end{abstract}

Keywords: digital ecosystem; information exchange; information sharing; reference architecture; theory of information trilogy

\section{Introduction}

Digital ecosystem (DE) is defined as "a set of distributed, adaptive, and open sociotechnical systems. Being parts of such ecosystems, individual persons, public and private organisations are becoming increasingly dependent on each other" [1]. DE, enabled by digital technology, is a complex network of individual and organisational actors (informational organism) who need to share information for value co-creation [2,3]. There are several types of DEs such as digital agriculture ecosystem, digital business ecosystem, digital government ecosystem, digital military ecosystem and digital health ecosystem etc. These DEs including physical ecosystems operate in the information sphere, which is also called "infosphere" [4]. Infosphere contains several informational entities or actors, which are called informational organisms. Information and its sharing (among informational organisms) are critical and core to these DEs. For instance, in a digital military ecosystem, efficient and effective information sharing is critical for coordinated decision making and operations in battlefields [5]. Furthermore, information sharing can also be observed in digital health [6] and government ecosystems [3].

There are several definitions of information [4,7]. The information concept can be defined as a set or group of related data elements including data contents and documents; thus, data are an implicit part of information [8]. Data elements represent raw facts encapsulated in data attributes, content containers, and documents [2,7]. The concept of information is relative and contextual in nature. For instance, it has been mentioned that "... the concept of information depends on the context of where and how it is being used" [9]. Thus, this paper aims to define information in the context of DE, which is inspired by natural ecosystems. Information management, from Information Science $[10,11]$ and Information Systems [12] disciplines, is an important capability that deals with the "control 
over how information is created, acquired, organized, stored, distributed, and used as a means of promoting, efficient and effective information access, processing, and use by people and organizations" [13]. A large amount of information or data are sourced from different channels and parties in DE [14]. Information sharing in DE is challenging [15,16]. This draws our attention to the following main research challenge and underpinning two research questions:

- Research Challenge: How to enable information sharing in complex DE?

- RQ1: What do we mean by information in a DE context?

- RQ2: How to share such information in DE?

This paper aims to address the above-mentioned research challenge and questions. The main contribution of this paper is two-fold: a theory of information trilogy (RQ1) and a conceptual model of DE information exchange architecture (RQ2). As indicated earlier, there are several definitions of information that describe information from storage, processing, management, probability, measurement, and quantification perspectives [17]. However, it is still not clear: What is information? How to identify it? Where to look for relevant information? Can it be destroyed? Can it be created? The proposed theory of information trilogy aims to address some of these ambiguities and provides the natural ecosystem-inspired definition of information, which states that "information is matter and energy, which has different states or forms. Matter and energy flow in the natural ecosystem".

This theory further explains how to translate these three concepts from a natural ecosystem to identify nine generic information elements (Table 1) and principles (Table 2), which can be used for identifying and handling information elements in complex DEs. In summary, this theory is defined with a view to define and identify information in complex and heterogenous DEs, which is a key contribution to the Information Science discipline. This theory also complements Shannon's mathematical theory of communication [18]. However, this theory can also be related to other important theories such as human information processing theory [19], no-hiding theorem [20], quantum information [21], the black hole information paradox [22], theory of relativity [23], laws of thermodynamics [24], and law of information conservation [25]. A detailed explanation of all these relevant theories is beyond the scope of this paper.

Table 1. Theory of information trilogy—generic information elements (based on [2]).

\begin{tabular}{ccc}
\hline Ref. & Information Element Type & Examples \\
\hline 1 & Organism & Individuals, organisations, animals, plants \\
2 & Place & Universe, country, state, city, suburb, road, building \\
3 & Time & Date and time \\
4 & Thing & Product, service, material, land, air, device \\
5 & Event & Sale, purchase, return, request \\
6 & Mechanism & Sell, buy, share \\
7 & State & Initiated, in progress, done \\
8 & Intuition & Emotions, feelings, beliefs, desires, intentions \\
9 & Consequence & Results, benefits, liability, value, responsibility \\
\hline
\end{tabular}

Once the information is defined and identified, then it can be shared in the DE. The "sharing" of information in DEs can be related to the concept of "flow" of matter and energy in natural ecosystems. Information sharing in complex and heterogenous DEs is not a simple task. This paper proposed the information exchange architecture model for enabling information sharing or flow in DE. This model can be used as a reference architecture to plan, architecture, and implement the specific digital information exchange platforms and systems involving both hardware and software. This model contributes to the Information Systems discipline. 
Table 2. Theory of information trilogy—generic information principles (based on [17]).

\begin{tabular}{cl}
\hline Ref. & \multicolumn{1}{c}{ Principle } \\
\hline 1 & Information presence can be realised when it takes us from an unknown to known \\
2 & state of matter or energy. \\
3 & Information is relative. \\
4 & Information is contextual. \\
5 & Information has a lifecycle. \\
6 & Information cannot be created. \\
7 & Information can be discovered. \\
8 & Information can emerge through interactions. \\
9 & Information is never lost. \\
\hline
\end{tabular}

It can be observed here that this research contributes to both Information Science and Systems disciplines and has implications for both theory and practice. This research aims to provide a conceptual foundation for setting a research and practice agenda in this emerging area of digital information exchange for information sharing in DE. The remainder of this paper is organised as follows. Firstly, it discusses the theory of information trilogy with a view to address RQ1, which provides the technology-independent definition of information in the context of DE. Secondly, it proposes the applied research-based practical and vendor neutral digital information exchange conceptual architecture for information sharing in DE. Finally, it concludes with key insights, contributions, and directions for future research.

\section{Theory of Information Trilogy}

There are several definitions of information and related concepts such as data, information itself, knowledge, and intelligence [4,7]. Information is sometimes called the new oil, soil, gold, etc. As indicated earlier, all these definitions and concepts seem useful from information storage, processing, and management perspectives. We still wonder what is "it", which we call "information". There is a need for defining the information in the current context of DE. The concept of DE is stirred from the natural ecosystem [2]. Thus, this paper proposes and describes a natural ecosystem-inspired definition of information for DE (RQ1), which is called here a "Theory of Information Trilogy" (ToIT) [17]. This theory defines that the "information is matter and energy, which has different states or forms. Matter and energy flow in the natural ecosystem" (see Figure 1). Furthermore, it provides nine generic information elements (Table 1) and principles (Table 2) for identifying and handling information in complex DEs. Building on this theory, information (matter, energy or state) flows (information sharing) in the DE. This theory is further elaborated in the following sub-sections.

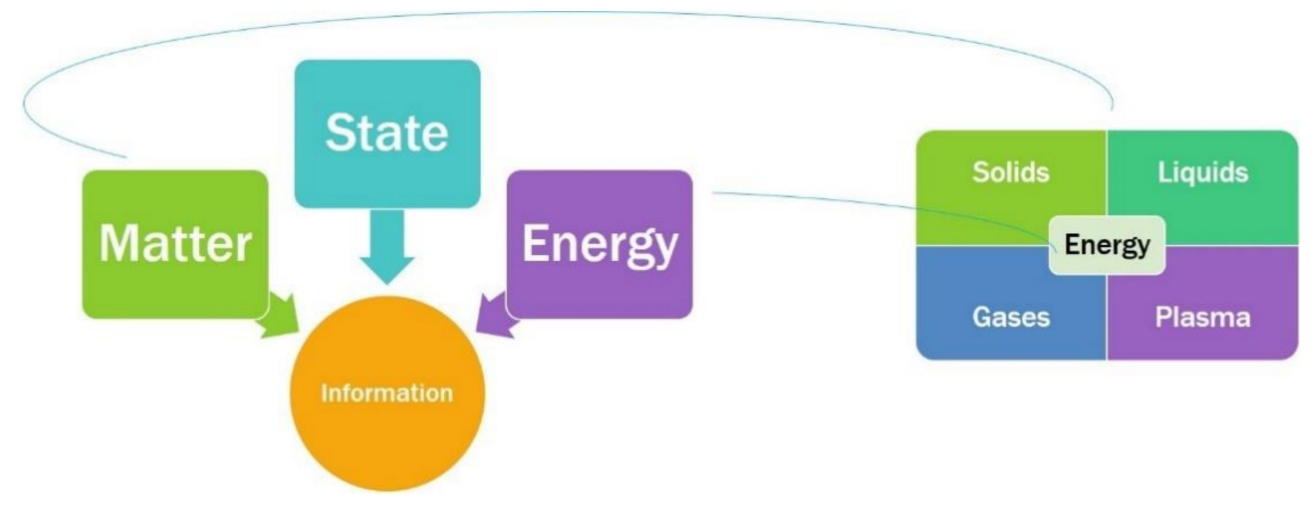

Figure 1. Theory of information trilogy—natural ecosystem view [17]. 


\subsection{Matter}

In a natural ecosystem, everything is information and information is life or a synonym for reality. We observe matter all around us in our natural ecosystem, which can be observed in different forms or states such as solid, liquid, gas, and plasma [26]. Take an example of a solid stone. Solid stone is a form or state of matter. Well, the question is then: what is "matter"? When we look at the stone, we detect its features such as size, weight, shape, colour, etc. What are we observing here? It is "information" that we are observing. Information is a set of relevant features. Thus, as a point of fact, solid stone is a visual representation of the information, which is visible in a solid form. The structure, behaviour, and purpose of this "form of information" can be further studied and analysed. Let us go back to our original question of: what is information? Take an example of an IoT oxygen sensor; it detects the presence of oxygen gas where gas represents the state of a matter (information). Thus, we may say that "information is or as a matter" is everywhere in our ecosystem and can be observed in different forms or states such as solids, liquids, gases, and plasma (see Figure 1). Then, physical information (matter) can be coded in a digital form to define the information for DE. Then, such digitised information can be shared among DE actors via DE information exchange, which will be discussed later in this paper.

\subsection{Energy}

Energy, similar to matter or information, also flows in our natural ecosystem. Energy is required to process things or carry out tasks. We need energy to detect matter, think, play, exercise, read, speak, breathe, smell, sense, etc. As per the law of conservation of energy [27], energy cannot be created or destroyed; however, it can be transferred from one form to another (e.g., chemical to electrical). Energy transformation and flow may involve matter, as per the laws of thermodynamics [24], such as information in each natural system, ecosystem, or DE (including surrounding environment). For instance, boiling water requires heat energy, a stove, and water in a water container. Energy in the form of heat energy will flow between the integrated and isolated system of matter (stove, water container, water) for obtaining hot water. Here, we can observe that energy is required to change the state of a matter (liquid) such as water from cold to hot water or steam. It is also important to note here that energy and its forms (e.g., chemical, electrical, gravitational, heat, light, mechanical energy, etc.) are also form of information. For instance, in the context of DE, information flows between actors (matter) to make decisions and perform certain tasks for a certain purpose. We may say that information (energy) interacts and flows in the DE. Furthermore, similar to energy, information (energy) cannot be destroyed or created (see law of conservation of energy). It has already been created and available in our ecosystem. It can be discovered. Once discovered, it can be combined, integrated, processed, and transformed to derive information and insights, which is different from creating information altogether from scratch.

We can conduct a simple experiment. Take a paper and ask someone (whom you do not know) to tell you, their name. You write their name on a piece of paper. After writing their name, you destroy the paper. Have you destroyed the information you just obtained (not created) and wrote on the paper? It seems that you have destroyed the information since you have destroyed the paper. Ask your brain about the name of the person whose name you just wrote on the destroyed paper. You may probably remember the name of the person and can reproduce on a paper (matter) or explain via verbal conversation or exchange (matter) using sound (energy). This explains us that information follows the law of conservation of energy. Information may be faded, which is not frequently accessed, but it is still somewhere there and can be reproduced or recycled. Furthermore, it can be observed that information emerges (is detected) when matter and energy (as information) interact with each other and flow in the DE. This draws our attention to the important insight that information can interact and flow in a DE during its exchange between the information holder and its audience. Furthermore, information such as energy cannot be destroyed; however, it can be transformed for a specific purpose. It also seems to follow 
relevant principles or laws of existence, form, and flow. Based on the analysis presented here, this paper reports on a set of generic information principles, which will be discussed later in this section.

\subsection{State}

Information, as a synonym for reality, is matter and energy, which may exist in different forms or states in a natural ecosystem and DE. These forms represent information (information model). Thus, these forms or states radiate information; therefore, they are also information. Thus, information is also a state of matter and energy, which takes matter or energy (information) from an unknown (unknown information) to a known (known information) state.

\subsection{Theory of Information Trilogy and Digital Ecosystem}

Information could be the new oil, soil, or gold that could be identified, collected, transformed, shared, and used. All these refer to the concept of matter, energy, and their forms or states. Hence, based on the analysis presented in the above-mentioned sections, in the context of a natural ecosystem-inspired system, it can be suggested that information is matter and energy, which can be recognised and flow in different forms or states (Figure 1) in DE. Information cannot be created and destroyed in DE; rather, it may be emerge through interactions between matter and energy. It can be discovered, transformed, shared, and consumed in DE (as per some principles or laws). The presence of information can be realised when it takes us from unknown (information debt or deficit) to a known state of something (information recognition) for a certain purpose. For the practical purpose and context of DE, concepts of matter, energy, and their states are translated to identify nine types of fundamental and generic information elements and principles [2,17].

\subsection{Theory of Information Trilogy and Generic Information Elements}

Generic information elements refer to organism, place, time, thing, event, mechanism, state, intuition, and consequence (Figure 2, Table 1). Digital information about these elements and their interactions can be shared in DE. Here, matter and energy are translated to form (state) an "organism". Thus, an organism is a combination of matter and energy and has different states. Furthermore, an organism could be human, animal, or plant. An organism is an ensemble of information (matter, energy, and state) and is a part of an infosphere (e.g., metaphysical and biosphere). If we focus on observing organisms (i.e., actors in DE) and their interactions, we can identify other elements of information: for example, organisms live in space (place) and time (data and time), they interact with things such as devices, etc. An approach centred on organism or actor interactions may help us identity the other eight elements (a compound of matter, energy, and state) (Figure 2, Table 1). These total nine generic elements, from the theory of trilogy, can be used as a lens and be instantiated for a specific type of DE (e.g., digital business ecosystem, digital financial ecosystem, digital health ecosystem) for identifying specific information elements, actors, and their interactions for further analysis, storage, and processing in the DE. Thus, it is possible that organisms could be interacting with each other and other things (equipment, devices) at a specific time and place (time and space). These organism interactions could be initiated or triggered by an event, which can then be executed via a specific mechanism (process). These organism interactions may lead to change in the current state of information. These organism interactions may also consider intuitions (e.g., emotions, feelings) and consequences (Table 1). There are several examples of organism interactions. For instance, there is an increasing use of social media by individuals and organisations (organisms or actors) to interact (interactions) with each other and share information [6]. Furthermore, interactions may involve asynchronous and synchronous modes of information sharing [3]. Well, it seems that information and its identification could be organism-interaction centric within the DE context [2]. Once information is identified using the nine types of generic 
information elements (Table 1), then it can be shared in a DE, which is further elaborated in the DE information exchange section.

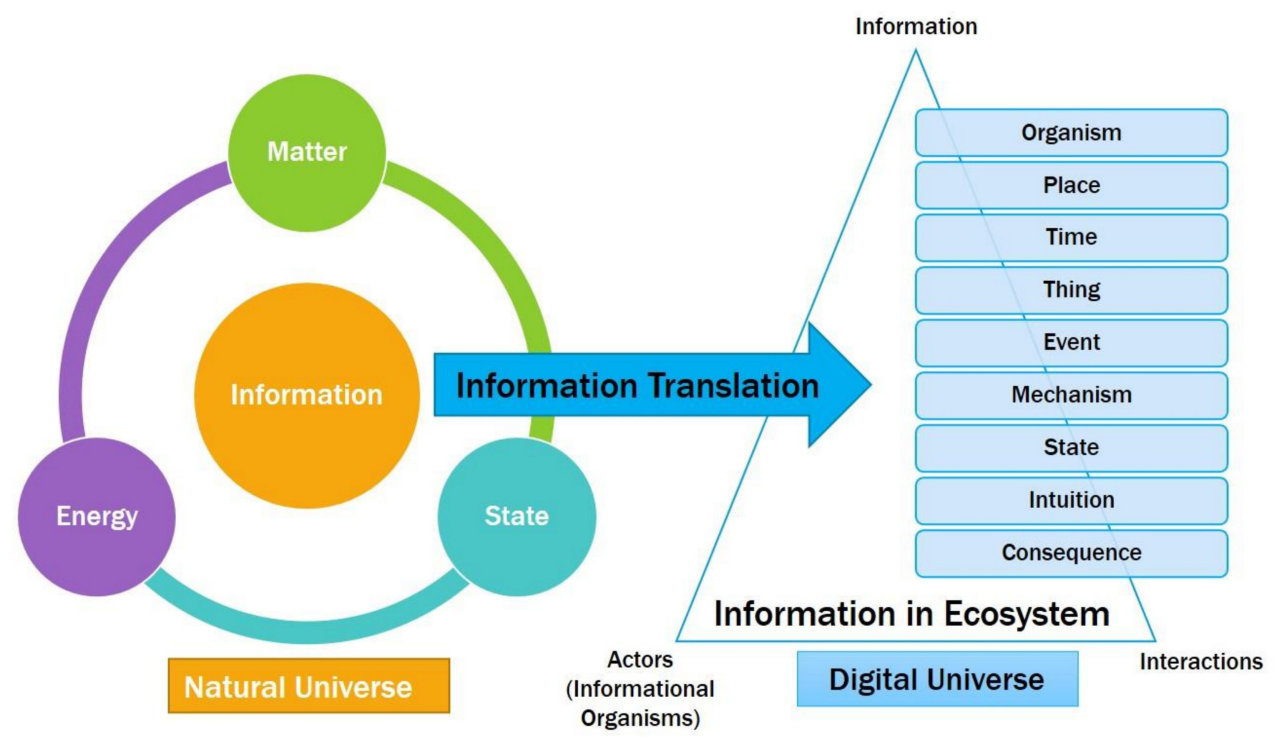

Figure 2. Theory of information trilogy_information ecosystem view (based on [2]).

\subsection{Theory of Information Trilogy and Generic Information Principles}

In addition to the above-mentioned nine generic information elements, the theory of information trilogy also provides nine generic information principles (Table 2) based on the study of a natural ecosystem and related theories (as indicated earlier). Both the generic information elements and principles can be used to design DE information architectures and systems. The first principle focuses on information recognition or presence based on the study of matter, energy, and their states in the natural ecosystem. The second principle mentions the relative nature of the information for a certain purpose (e.g., information value, scale, meaning). The third principle indicates the contextual nature of the information (e.g., data in each context). The fourth principle suggests that information has a lifecycle similar to other natural elements (e.g., discover and collect, classify and secure, prepare and release, access and use, archive and purge, restore and recycle). The fifth and ninth principles indicate that information is conserved or protected; thus, it cannot be created and lost (based on the law of energy and information conservation). However, the sixth principle indicates that information can be discovered. Following this, the seventh principle suggests that information can emerge through interactions. Thus, to identify information, we need to study informational organisms and their interactions (as mentioned earlier). The eighth principle indicates that information can be translated from one form to another form, which is different from creating information. These principles can be adapted for DEs.

The theory of information trilogy elements and principles can be further explored with the mathematical theory of communication [18], human information processing theory [19], no-hiding theorem [20], quantum information [21], the black hole information paradox [22], theory of relativity [23], laws of thermodynamics [24], and law of information conservation [25].

\section{Digital Ecosystem Information Exchange}

Traditional approaches of point-to-point and ad hoc information integrations hinder the ability to share different types of information at a large scale between different types of large number of actors operating in the DE [1]. The information-sharing landscape has evolved and is continuously evolving from traditional electronic data interchange (EDI) and file transfer protocols (FTP) to contemporary cloud-based APIs and solutions (OneDrive, Google Drive, Amazon S3, Azure Blob Storage, Google Cloud Storage), etc. 
Furthermore, sourcing, integrating, and sharing information to/from different actors, in different sizes and formats, via multiple channels and across jurisdictions in a DE is not only difficult; rather, sometimes, it is not achievable. There is a need for a seamless and plug-and-play kind of adaptable secure information-sharing architecture and platform. This section proposes one such digital information exchange architecture (see Figure 3) as an emerging concept for the seamless, effective, efficient, and adaptable secure information sharing among different DE actors (RQ2). The focus is not to create another centralised data hub or data marketplace as a honey pot for hackers. However, a centralised data hub or data marketplace can be connected to the information exchange platform either as a source or destination of information. Thus, a user may source and publish information to/from a data hub or data marketplace. The proposed digital information exchange architecture is underpinned by design principles of logical information or data abstraction, decoupling, federation, interoperability, resiliency, and virtualisation.

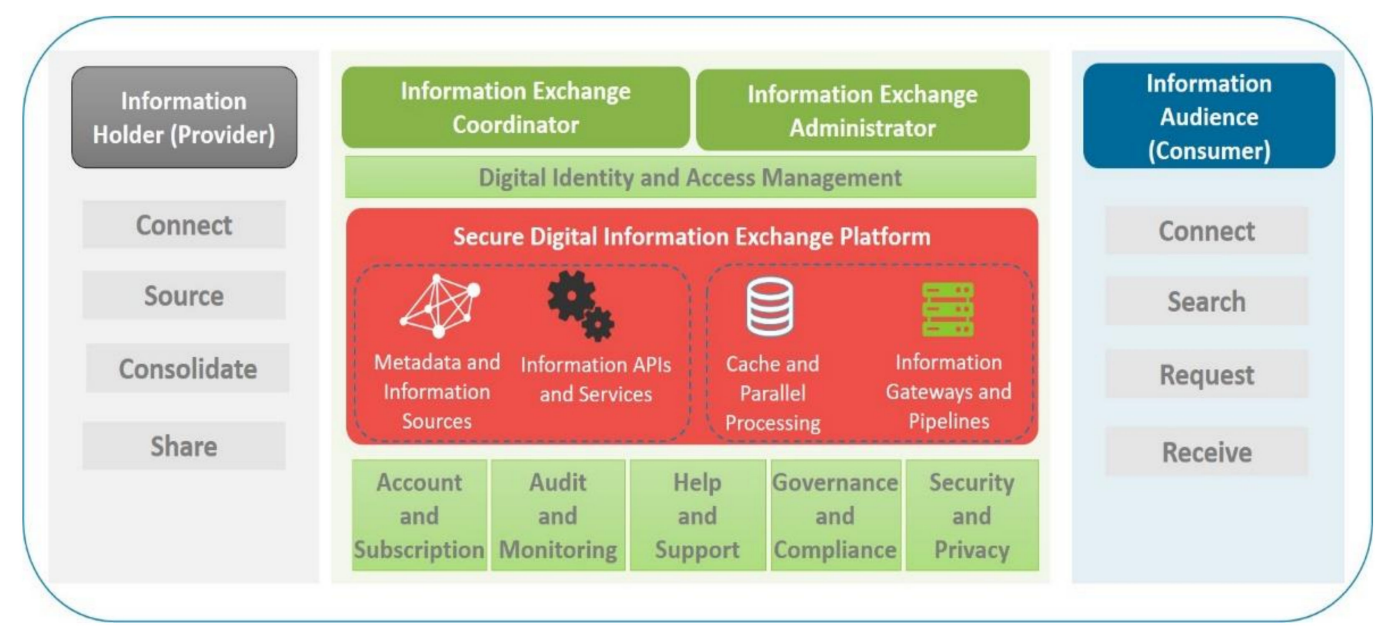

Figure 3. Digital information exchange: a conceptual architecture.

\subsection{Digital Information Exchange}

The organism-interaction centric DE information exchange architecture has three key components: actors (informational organisms), services (interactions), and a platform (Figure 3). The digital information exchange enables the information holder (provider) actors to share the information (outbound), including allowable privileges and conditions, with the intended audience (information consumer) via connect, source, consolidate and share services. Information audience (consumer) actors can access available information (inbound), including allowable privileges and conditions, via connect, search, request and receive services. It is important to note here that a consumer's use of the inbound information is controlled by the allowable privileges and conditions set by the sharer (provider). Information sharing is overseen and facilitated by the information exchange coordinator via governance and compliance services (e.g., coordinating steward). An information exchange administrator manages the exchange operations using core operational and business support services: digital identity and access management, audit and monitoring, account and subscription, help and support, and security and privacy.

\subsubsection{Information Holder}

An information holder can access the information exchange platform APIs and services via a digital identity and the access management layer. These APIs and services enable the user to connect with the secure digital information exchange platform and then source, consolidate, and share information with the intended audience in different formats. The following are core APIs and services for the information holder. 
- Connect: It allows connecting with the secure information exchange platform using their account. This could be done via protected devices, connections, applications, and networks.

- Source: It involves selecting and virtualising the required information source including validation and fixing any information quality issues.

- Consolidate: It involves the consolidation of information sourced from different sources before sharing. Consolidation also involves the mapping, merging, and transformation of information for the intended audience. Information, which is subject to sharing, could be reference data, master data, metadata, transactional data, or insights.

- Share: It involves packaging and dispatching consolidated information to their intended audience in different forms via listed or published information APIs and services. Information APIs and services allow the provider to provide "information as code". Thus, rather than sharing the actual information, only a code or script can be shared, which can be executed by the receiver to retrieve the information package. A secure outbound information package contains information objects (e.g., metadata, schema, tables, views, records, insights), including privileges and conditions. A secure outbound information package is accessible via information APIs and services. Privileges and conditions determine the further shareability of the shared information. It means consumers may not be able to share the shared information. This allows the information owners to have control over their information even after it has been shared with the intended audience or consumers. Furthermore, information could be directly and privately shared with an intended consumer based on information request or could be listed and privately or publicly made available to multiple consumers via the marketplace.

\subsubsection{Information Audience}

The information audience (consumer) can access the information exchange platform APIs and services via a digital identity and the access management layer. These APIs and services enable the information audience to connect with the secure digital information exchange platform and search, request, and receive information from the information holder. The following are core APIs and services for the information audience.

- Connect: It allows connecting with the secure information exchange platform using their account. As indicated earlier, this could be done via protected devices, connections, applications, and networks.

- Search: It allows searching metadata and information sources including information APIs and services (catalogues) available for sharing.

- Request: It allows the intended audience to request information from the information holder using multiple channels (e.g., email, instant message, API, or service calls).

- Receive: It enables the intended audience to receive shared information (inbound) in different forms (e.g., information APIs, services) from the information holder. The audience can access and use the shared information as per allowed privileges and conditions. Information could be received in real time, in batches, etc. The shared information can be combined with other information sources for rich analytics and insights.

\subsubsection{Information Exchange Coordinator and Administrator}

Information exchange coordinators (e.g., stewards) and administrators (e.g., system, account, support, security administrator) are responsible for the smooth operations, management, and governance of information sharing and the information exchange platform. This is done via the following key enabling services.

- Digital identity and access management: This service, including privileged access management, allows (platform system administrator) creating users, verifying digital 
identity, and controlling access and permissions (e.g., create, read, update, delete, share) to the DE information exchange platform and the underlying information APIs.

- Account and subscription management: This service enables (platform account administrator) managing account onboarding, subscriptions, information sharing contracts, transactions, history, payments, and feedback related to DE information exchange platform and its information APIs. This involves overall account and subscription information management, reporting, and analytics.

- Audit and monitoring: This service enables (platform system administrator) auditing and monitoring information lineage and sharing via the DE information exchange platform. This involves overall audit and monitoring logs management, reporting, and analytics.

- Help and support: This service provides (platform support administrator) operational support, trouble shooting, and requested information to DE information exchange platform users.

- Governance and compliance: This service ensures (information exchange coordinator or steward) the overall governance of the information sharing and DE information exchange platform, and its compliance to different information laws, regulations, standards, and principles. This also ensures that the information is of good quality and is fit for purpose. Information, subject to sharing, could be pre-qualified and certified as per specific context and needs.

- Security and privacy: This service ensures (platform security administrator) the security and privacy of information and a DE information exchange platform for smooth, secure, and resilient operations.

\subsubsection{Information Exchange Platform}

The DE information exchange platform enables the sharing of digital information between different actors operating in the DE. Thus, the focus is on "secure digital information exchange". Unlike traditional centralised data lakes, hubs or marketplace architectures, exchange platform architecture does not store the actual information. It focuses on the storage minimisation principle. It enables information sharing via cached federated information sources and the virtualisation approach. This approach provides a logical metadata-driven virtual abstraction layer, which is decoupled from the actual physical information and its source. This provides access to information, which is kept at its source or origin. This avoids the unnecessary need for moving, copying, or duplicating information (for sharing purposes) in a central store or marketplace. The following are the key components of the information exchange platform.

- Metadata and information sources: The DE information exchange mainly stores the metadata about information and its sources. Metadata could be in the form of information design metadata (e.g., information definitions, models, table structures, schemas), information execution metadata (e.g., information processing logs, scripts) and actual information usage metadata (e.g., information access and usage patterns, experience). Information sources could be local or cloud-based information stores, warehouses, data lakes, IoT, APIs, services, etc. Here, the focus is to virtualise the information and its sources rather storing the information in a central data hub or marketplace.

- Information APIs and services: Information and its sources are exposed via information APIs and services. Thus, actors do not have direct access to information and its sources. These APIs and services can be used by other APIs or services or applications (e.g., Amazon, Azure, Google Cloud, Power BI, Tableau). They can also be used to develop specific applications using scripting or programming languages (e.g., Java, Python, Node.js, .Net, SQL). These APIs and services should be accessible via secure shell or web browsers. These APIs and services need memory and computing resources such as cache and parallel processing for execution. 
- Cache and parallel processing: Information and its sources can be cached for optimising performance and resiliency. This enables the ability to provide information from cache memory to users when the physical information source is not available or down. Furthermore, parallel information processing can be achieved to handle a large amount of information. Cached memory and parallel information processing could come in different sizes (e.g., small, medium, large, extra-large) and have the autoscaling features including clustering of resources (e.g., scaling-in, scaling-out, scaling-up, and scaling-down). Cache and parallel processing provide essential fast memory and computing components for information exchange. Cache and parallel processing nodes can be connected via information gateways and pipelines.

- Information gateways and pipelines: The DE information exchange needs to support multiple gateways to allow the user to connect with the DE information exchange pipelines via different interfaces and locations. Information gateways and pipelines provide essential secure connectivity and network components for information exchange. Information pipelines could be open public, protected, or private (sensitivity and criticality) and could also be classified as cold, warm, and hot pipelines depending on the nature of information (e.g., real-time, recent, historical, and archived information). In summary, information pipelines enable the secure transfer of information from its sources to intended destination (Figure 4) — see the mathematical theory of communication by Shannon [18].

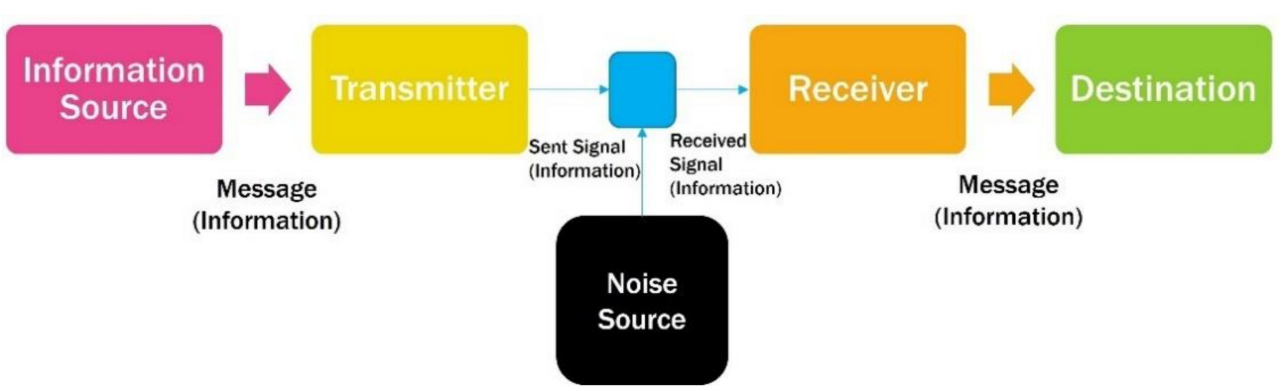

Figure 4. General information pipeline-mathematical theory of communication [18].

In a nutshell, DE information exchange provides a secure and cached federated architecture for sharing information between multiple actors operating in the distributed and open DE. There are several ways to deploy digital information exchanges, which are discussed in the following section.

\subsection{Digital Information Exchange Deployment Models}

There are four key digital information exchange deployment models: public, protected, private, and hybrid digital information exchange. A public digital information exchange is deployed for sharing information, which is open to the public. This exchange model can be established at the local and national level to support the DE actors i.e., individuals and organisations. A protected digital information exchange is established to share "protected information" with trusted users. A private digital information exchange is internal to an organisation and only focuses on the sharing of information between internal users. A hybrid model of integrated public, protected, and private exchanges can also be used as appropriate to specific context and needs. Thus, a private internal exchange can be connected to public or protected digital information exchanges as appropriate to the context and information sharing needs. Furthermore, these exchanges could be fixed or deployable on demand at different remote locations or sites.

\section{Geospatial Information Sharing Case Study}

This section describes the applicability of the digital information exchange architecture to a geospatial information sharing case study example. This case study example is based 
on the real-world emergency management scenario. Emergency management requires a multi-agency response and involves several actors (e.g., ambulance, fire, emergency department, police, volunteers) to handle emergencies (e.g., floods, earthquakes). These agencies or actors need to share large amount of geospatial information (e.g., location of critical assets such as electrical systems, communication lines, water lines, transport, schools, hospitals, evacuation points) to support the planning and coordination of activities involving emergency workers and volunteers [28]. Point-to-point or peer-to-peer information sharing (from geospatial information holders to intended audience) is cumbersome in this situation and is not only difficult, but it is impossible to achieve where a quick response is required to handle emergencies.

It is critical to get the right information to the right people at the right for efficient and effective decision making and actions [29]. An on-demand deployable protected digital emergency information sharing exchange can be deployed, as proposed in the passing section, to allow the agencies and volunteers to share geospatial information in a trusted manner beyond the boundary of a single organisation (see Figure 5). A chief information coordinator or steward can be appointed from a coordinating agency (e.g., police or state emergency services) to oversee and coordinate the information sharing from geospatial information holders to an intended audience such as ambulance, fire, and volunteers for planning and coordinating emergency management activities. Once an emergency is resolved, then the protected digital emergency information sharing exchange can be rolled back (undeployed), and necessary information, if any, from the exchange is exported to relevant agencies for future planning and reference. A deployable protected information exchange seems to be a cost-effective and agile solution for information sharing among trusted parties, which can be deployed (spin-up) and undeployed (spin-down) on demand in the cloud [30].

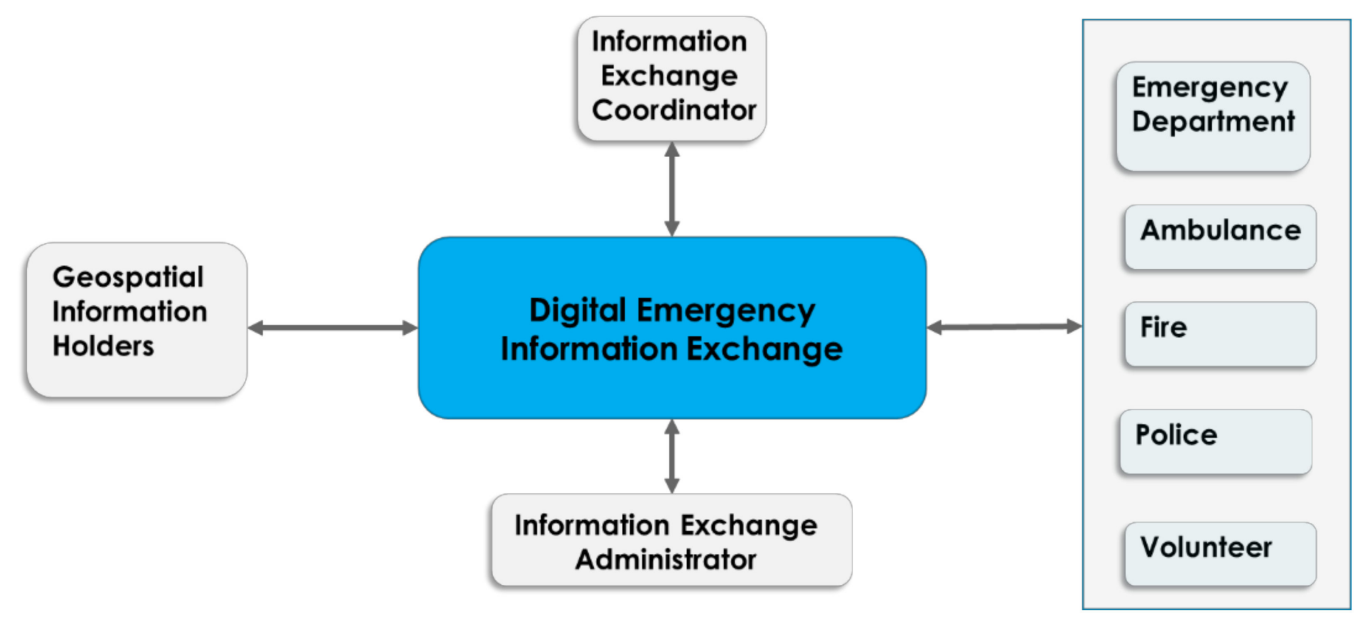

Figure 5. Deployable digital emergency information exchange.

\section{Conclusions, Contribution, and Future Research Directions}

Information sharing is challenging in DE. However, before discussing the information sharing, it is important to unpack the important concept of information for DE. This paper defined a natural ecosystem-inspired definition of "information" for DE, which is independent of its storage, processing, and related technologies (data hubs, lakes, data warehouses etc.). This paper discussed a theory of information trilogy involving three key concepts of matter, energy, and their forms or states, and nine generic information elements and principles. It considers that "information is matter and energy, which has different states or forms. Matter and energy flow in the natural ecosystem". Thus, it is called a "Theory of Information Trilogy". This theory complements the mathematical theory of communication [18], which mainly focuses on the communication of the information from source to destination. It does not seem to focus on the information itself. Thus, the theory 
of information trilogy aims to address this gap and defines the information element and its flow in the natural context of ecosystem, which is then translated to information sharing in DE. Both the integrated theory of information trilogy and the mathematical theory of communication can be used to study and model digital information and its sharing in DE. It has been noted here that the mathematical theory of communication seems useful for modelling digital information pipelines as mentioned in the DE information exchange architecture (Figure 4).

Once the information is identified and understood, then it can be shared in DE. This draws our attention to the second contribution of this paper, which is the proposed conceptual model of the DE information exchange architecture. The applicability of the proposed model is demonstrated with the help of a geospatial information sharing example scenario. The proposed information exchange conceptual model seems useful for information sharing. This seems to resolve peer-to-peer and ad hoc information sharing pain points. This conceptual model also uncovers the areas of further research. For instance, further research is required to understand the following key questions:

- How can we design a distributed digital information exchange organisational structure?

- How can we ensure information governance, privacy, and compliance across different local, national, and international jurisdictions?

- How should we choose between and design appropriate digital information exchange deployment models?

- How can we on-board and off-board users, their metadata, and information sources?

- How should we price digital information exchange APIs and services?

- How can we integrate digital information exchanges with the existing internal information infrastructure and environments (data sources, data hubs, data warehouses, data lakes, staging areas, consumption areas)?

This paper provided a conceptual foundation of information and digital information exchange architecture for information sharing in DE. Similar to any other research, this research has limitations. Firstly, there is a lack of published academic research in this emerging topic of digital information exchange architecture, which limited the ability to test and compare the proposed model against the established models or standards. Secondly, results from a single case study cannot be generalised. Thus, this study can be further enhanced through empirical research in different types of DEs. Thirdly, this paper mainly discussed and assumed the normal matter and energy. This research can be expanded to study dark matter and energy, which are difficult to detect in the natural ecosystem. The learnings from the study of dark matter and dark energy can be translated to identify dark data or information [31] in DEs (e.g., unstructured big data and information stores) [32]. Finally, this research does not provide digital information exchange architecture implementation method or guidelines, which can be further researched and shared with the community in future. Nevertheless, the proposed theory and model provide a conceptual foundation for advancing knowledge in both Information Science and Information Systems disciplines. it is anticipated that learning from nature is useful [33] when dealing with information to alleviate our information and related pain points for desired aspirations and goals of information sharing. Future research will investigate and study the natural ecosystems with a view to some of the above-mentioned research questions.

Funding: This research received no external funding.

Institutional Review Board Statement: Not applicable.

Informed Consent Statement: Not applicable.

Data Availability Statement: Not applicable.

Acknowledgments: I would like to extend my sincere thanks to several colleagues from academia and industry who provided me valuable feedback over a period of several years to shape the work presented in this paper. 
Conflicts of Interest: The author declares no conflict of interest.

\section{References}

1. Krogstie, J. Modeling of Digital Ecosystems: Challenges and Opportunities. In Collaborative Networks in the Internet of Services; PRO-VE 2012; IFIP Advances in Information and Communication Technology; Camarinha-Matos, L.M., Xu, L., Afsarmanesh, H., Eds.; Springer: Berlin/Heidelberg, Germany, 2012; Volume 380. [CrossRef]

2. Gill, A.Q. Adaptive Information Architecture: Information Element Identification and Organisation Patterns for Digital Ecosystem. In Proceedings of the Information and Knowledge Engineering Conference, CSCE, Las Vegas, NV, USA, 26-29 July 2021.

3. Alam, L.; Gill, A. A Social Engagement Framework for the Government Ecosystem: Insights from Australian Government Facebook Pages. International Conference on Information Systems. 2020. Available online: https://aisel.aisnet.org/icis2020/ digitization_in_cities/digitization_in_cities/8 (accessed on 16 July 2021).

4. Floridi, L. Information: A Very Short Introduction; Oxford Press: Oxford, UK, 2010.

5. Farooq, M.J.; Zhu, Q. Secure and reconfigurable network design for critical information dissemination in the Internet of battlefield things (IoBT). In Proceedings of the 15th International Symposium on Modeling and Optimization in Mobile, Ad Hoc, and Wireless Networks (WiOpt), Paris, France, 15-19 May 2017; pp. 1-8. [CrossRef]

6. Lin, H.C.; Chang, C.M. What motivates health information exchange in social media? The roles of the social cognitive theory and perceived interactivity. Inf. Manag. 2018, 55, 771-780. [CrossRef]

7. Liew, A. Data, information, knowledge, and their interrelationships. J. Knowl. Manag. Pract. 2007, 7, 2.

8. Earl, M.J. Management Strategies for Information Technology; Prentice Hall: New York, NY, USA; London, UK, 1989.

9. Logan, R.K. What Is Information? Why Is It Relativistic and What Is Its Relationship to Materiality, Meaning and Organization. Information 2012, 3, 68-91. [CrossRef]

10. Borko, H. Information science: What is it? Am. Doc. 1968, 19, 3. [CrossRef]

11. Saracevic, T. Information science. In Encyclopedia of Library and Information Sciences, 3rd ed.; Bates, M.J., Ed.; Taylor and Francis: New York, NY, USA, 2009; pp. 570-2585.

12. Davis, G.B. Information Systems as an Academic Discipline. In The Past and Future of Information Systems: $1976-2006$ and Beyond; IFIP WCC TC8 2006; IFIP International Federation for Information Processing; Avison, D., Elliot, S., Krogstie, J., Pries-Heje, J., Eds.; Springer: Boston, MA, USA, 2006; Volume 214, pp. 11-25. [CrossRef]

13. Detlor, B. Information management. Int. J. Inf. Manag. 2010, 103-108. [CrossRef]

14. Bashir, M.R.; Gill, A.Q. IoT enabled smart buildings: A systematic review. IEEE Intell. Syst. Conf. 2017, 151-159. [CrossRef]

15. Chessell, M.; Smith, H.C. Patterns of Information Management; IBM Press: Armonk, NY, USA, 2013.

16. Sharma, A.; Rana, N.P.; Nunkoo, R. Fifty years of information management research: A conceptual structure analysis using structural topic modeling. Int. J. Inf. Manag. 2021, 58. [CrossRef]

17. Gill, A.Q. What Is Information? A Theory of Information Trilogy. Available online: https://aqgill.blogspot.com/2021/06/whatis-information-theory-of.html (accessed on 14 June 2021).

18. Shannon, C.E. A Mathematical Theory of Communication. Bell Syst. Tech. J. 1948, 27, 379-423. [CrossRef]

19. Atkinson, R.C.; Shiffrin, R.M. Human Memory: A Proposed System and Its Control Processes. In Human Memory: Basic Processes; Elsevier: Amsterdam, The Netherlands, 1977; pp. 7-113. [CrossRef]

20. Braunstein, S.L.; Pati, A.K. Quantum Information Cannot Be Completely Hidden in Correlations: Implications for the Black-Hole Information Paradox. Phys. Rev. Lett. 2007, 98, 8. [CrossRef] [PubMed]

21. Nielsen, M.A.; Chuang, I.L. Quantum Computation and Quantum Information, 10th Anniversary ed.; Cambridge University Press: Cambridge, UK, 2012.

22. Musser, G. The Most Famous Paradox in Physics Nears Its End. 2020. Available online: https://www.quantamagazine.org/theblack-hole-information-paradox-comes-to-an-end-20201029/ (accessed on 14 July 2021).

23. Einstein, A. Relativity: The Special and General Theory; H. Holt and Company: New York, NY, USA, 1916.

24. Guggenheim, E.A. Thermodynamics: An Advanced Treatment for Chemists and Physicists, 11th ed.; Zeitschrift für Naturforschung: Amsterdam, The Netherlands, 1985.

25. Zhang, B.; Cai, Q.; Zhan, M.; You, L. Information conservation is fundamental: Recovering the lost information in Hawking radiation. Int. J. Mod. Phys. D 2013, 22, 12. [CrossRef]

26. Britanica. Matter. Available online: https://www.britannica.com/science/matter (accessed on 14 June 2021).

27. Feynman, R. The Feynman Lectures on Physics Vol I; Addison Wesley: Boston, MA, USA, 1970; ISBN 978-0-201-02115-8.

28. Bhanumurthy, V.; Shankar, G.J.; Rao, K.R.M.; Nagamani, P.V. Defining a framework for integration of geospatial technologies for emergency management. Geocarto Int. 2015, 30, 963-983. [CrossRef]

29. Gill, A.Q.; Phennel, N.; Lane, D.; Phung, V.L. IoT-enabled emergency information supply chain architecture for elderly people: The Australian context. Inf. Syst. 2016, 58, 75-86. [CrossRef]

30. Štefanič, P.; Cigale, M.; Jones, A.C.; Knight, L.; Taylor, I.; Istrate, C.; Suciu, G.; Ulisses, A.; Stankovski, V.; Taherizadeh, S.; et al. SWITCH workbench: A novel approach for the development and deployment of time-critical microservice-based cloud-native applications. Future Gener. Comput. Syst. 2019, 99, 197-212. [CrossRef]

31. Grimm, D.J. The Dark Data Quandary. Am. Univ. Law Rev. 2019, 68, 761-821. Available online: https://digitalcommons.wcl american.edu/aulr/vol68/iss3/2 (accessed on 16 July 2021). [PubMed] 
32. Anwar, M.J.; Gill, A.Q.; Hussain, F.K.; Imran, M. Secure big data ecosystem architecture: Challenges and solutions. EURASIP J. Wire 2021. [CrossRef]

33. Luhn, G. The Causal-Compositional Concept of Information Part I. Elementary Theory: From Decompositional Physics to Compositional Information. Information 2012, 3, 151-174. [CrossRef] 